\title{
Unusual cytological finding of sheathed microfilariae in a fine needle aspirate: a case report
}

\author{
P. Preena ${ }^{{ }^{*}}$, Sherin B. Sarangom ${ }^{2}$ and Seeja Sundaran ${ }^{2}$ \\ District Veterinary Centre, Kannur, \\ Department of Animal Husbandry, Kerala, India
}

Citation: Preena, P., Sherin, B. S. and Seeja, S. 2021. Unusual cytological finding of sheathed microfilariae in a fine needle aspirate: A case report. J. Vet. Anim. Sci. 52(1): 92-94.

DOI: https://doi.org/10.51966/jvas.2021.52.1.92-94

Received:01.08.2020

17.08.2020

Published:01.01.2021

Filariasis is a neglected mosquito-borne tropical parasitic disease with public health importance in tropical and subtropical countries. Canine filarial infections are caused by many nematode species such as Dirofilaria immitis, D. repens, Brugia pahangi, B. malayi and Acanthocheilonema reconditum (Satjawongvanit et al., 2019). Microfilariae of Brugia spp. are sheathed while those of others are non-sheathed (Ravindran et al., 2014). Canine filarial infections are highly endemic to the state of Kerala with the reports of prevalence of microfilaraemia ranging from 8.1 per cent in Thrissur district to very high prevalence of 42.68 per cent in Alappuzha district (Chirayath et al., 2017; Ravindran et al., 2014). The major clinical signs in dogs associated with circulating microfilariae are fever, anorexia, conjunctivitis, dermatitis, oedema of limb and scrotum (Tarello, 2011). The traditional methods for identification of the microfilariae are based on morphological microscopic observation of Giemsa stained peripheral blood smears. Despite the high prevalence of filarial infections in dogs, it is infrequent to find the microfilariae in fine needle aspiration cytology (FNAC) in suspected cases of limb oedema. The demonstration of microfilariae in the aspirate or body fluids plays a pivotal role in diagnosis of the condition and institution of specific treatment modalities, especially in case of lymphatic filariasis (Panicker et al., 2012). In the present communication, a case of canine filarial infection with right forelimb oedema, chronic nonhealing ulcers and localized alopecia diagnosed based on fine needle aspiration cytology ( FNAC) is discussed.

A seven-year old female Labrador dog (Fig. 1A) was presented at the Out Patient Ward of the District Veterinary Centre, Kannur (Dist), Kerala with complaints of oedema and wounds on the right forelimb (Fig. 1B). Whole blood ( $2 \mathrm{~mL})$ was collected from the saphenous vein for complete blood count (CBC) using automated hematology analyzer (Exigo EOS, Sweden). Peripheral blood from the tip of the ear was collected for wet film examination and for making blood smears for microscopic examination after Giemsa staining. Skin scrapings were collected from the lesions in the oedematous area for parasitological examination. Fine needle aspirate and impression smears were also collected from the oedematous area of the limb for parasitological and cytological examinations. Hematological analysis revealed moderate regenerative anaemia with a haemoglobin level of $9.4 \mathrm{~g} / \mathrm{dL}$, total RBC count of $4.5 \mathrm{million} / \mathrm{cu} . \mathrm{mm}$ and haematocrit of 28 per cent.

1. Veterinary Surgeon and corresponding author, email id: preenalinesh@gmail.com

2. Veterinary Surgeons

Copyright: () 2021 Preena et al. This is an open access article distributed under the terms of the Creative Commons Attribution 4.0 International License (http://creativecommons.org/licenses/by/4.0/), which permits unrestricted use, distribution, and reproduction in any medium, provided the original author and source are credited. 
On wet film examination, numerous live and motile microfilariae were detected exhibiting snake like wriggling movement across the microscopic field (average of 6 organisms per 10X field). No ectoparasites were detected in the skin scrapings collected, while FNAC and impression smears revealed moderately cellular smears with neutrophils and multiple (sheathed) microfilaria on a haemorrhagic background (Fig. 2A). Giemsa stained blood smears on microscopical examination revealed numerous polychromatophils, a few toxic neutrophils and sheathed microfilariae. Microfilariae showed a bluish violet coloured body and a pink coloured sheath extending beyond the anterior and posterior ends. The anterior end was blunt with the cephalic space devoid of any nuclei, darkly stained tightly packed nuclear column and the posterior end with two distinctly separated terminal nuclei at the tail tip (Fig. 2B). Presence of sheath and two distinct widely separated terminal nuclei confirmed the Brugia spp.

Based on cytological and hematological evidences, levamisole @ 10mg/kg OD per orally for one week, doxycycline @ $10 \mathrm{mg} / \mathrm{kg}$ per orally for two weeks, pantoprazole @ $1 \mathrm{mg} /$ $\mathrm{kg}$ per orally before food for two weeks were administered. In addition, liver tonics and an antiseptic wound spray were also prescribed for two weeks. Considerable improvement was noticed after two weeks of treatment. There was a marked reduction in oedema and swelling along with healing of the ulcerated wounds. No microfilariae could be detected at this stage in FNAC. In addition, numerous degenerated neutrophils, plasma cells, eosinophils and neutrophils engulfing hemosiderin pigments were also noticed in FNAC. Panicker et al. (2012) stated that dead or fixed worms and microfilaria can excite severe reaction leading to eosinophilia, eosinophilic abscess, necrosis, fibrosis and epithelioid granuloma.

Previous studies had diagnosed most of the cutaneous and subcutaneous masses in dogs as being due to infection with $D$. repens (Abd Rani et al., 2010). Thus from the present study, it could be concluded that the presence of sheathed microfilariae in the fine needle aspirate and impression smears can be used

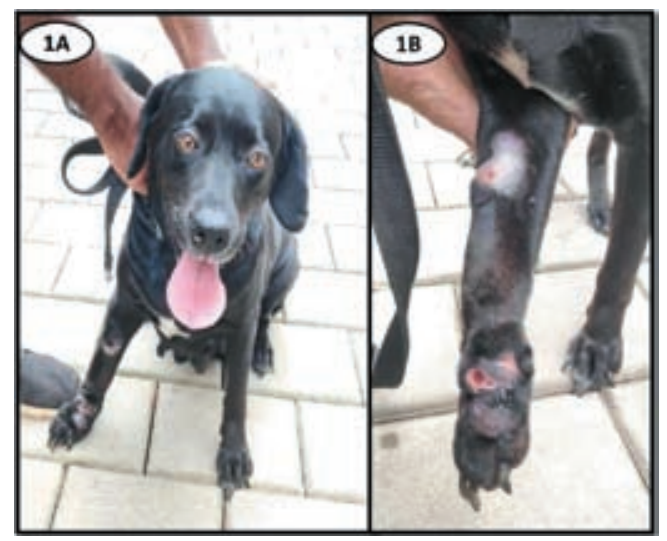

Fig. 1A: The dog presented with limb oedema \& localized alopecia; 1B: The edematous right forelimb with ulcerated non-healing wounds could be noted

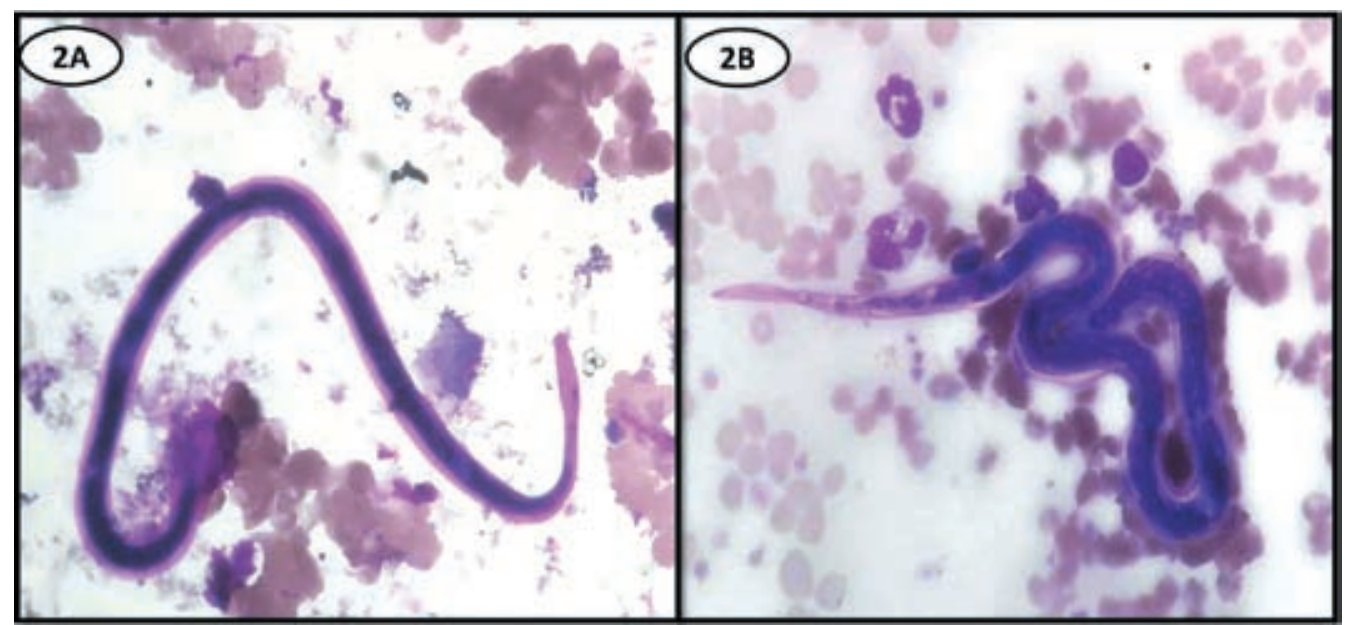

Fig 2A: Presence of sheathed microfilaria in the fine needle aspirate of edematous limb;2B: Presence of sheath and two distinct widely separated terminal nuclei of the microfilaria in the blood smear may be noted 
as a method for diagnosis of lymphatic filarial infections of dogs. For confirmation of the filarial species, molecular techniques are essential.

\section{SUMMARY}

Fine needle aspiration cytology was used to confirm the presence of Brugia spp. infection in dogs causing limb oedema and dermatopathies. Clinicians mustcarefully consider this diagnostic possibility when dealing with similar cases.

\section{REFERENCE}

Abd Rani, P.A.M., Irwin, P.J., Gatne, M., Coleman, G.T., Mclnnes, L.M. and Traub, R.J. 2010. A survey of canine filarial diseases of veterinary and public health significance in India. Parasit. Vectors. 3(1): 30.

Chirayath, D., Alex, P.C., Usha, N.P., George, S., Ajithkumar, S. and Panicker V.P. 2017. Identification of Brugia malayi in dogs in Kerala, India. Trop. Biomed. 34: 804-814.

Panicker, N.K., Buch, A.C., Vimal, S. and Dharwadkar, A.P. 2012. Cytological diagnosis of microfilariae in subcutaneous nodule. Med. J. DY Patil University. 5(1):71.

Ravindran, R., Varghese, S., Nair, S.N., Balan, V.M., Lakshmanan, B., Ashruf, R.M., Kumar, S.S., Gopalan, A.K.K., Nair, A.S., Malayil, A. and Chandrasekhar, L. 2014. Canine filarial infections in a human Brugia malayi endemic area of India. BioMed Res. Int. 2014: 630160.

Satjawongvanit, H., Phumee, A., Tiawsirisup, S., Sungpradit, S., Brownell, N., Siriyasatien, P. and Preativatanyou, K. 2019. Molecular analysis of canine filaria and its Wolbachia endosymbionts in domestic dogs collected from two animal university hospitals in Bangkok Metropolitan Region, Thailand. Pathogens. 8(3): 114.

Tarello, W. 2011. Clinical aspects of dermatitis associated with Dirofilaria repens in pets: a review of 100 canine and 31 feline cases (1990-2010) and a report of a new clinic case imported from Italy to Dubai. J. Parasitol. Res. 2011:578385. 